\title{
COMPUTED TOMOGRAPHY OF MALIGNANT TUMORS OF THE OSSEOUS PELVIS
}

\author{
Marco A. Amendola ${ }^{1}$, Khalil Shirazi ${ }^{1}$, Beatriz E. Amendola', \\ LAWRENCE R. KUHNS ${ }^{1}$, JAIME TISNADO ${ }^{2}$ and ISSA YAGHMAI ${ }^{2}$ \\ 'Department of Radiology, University of Michigan Medical Center, Ann Arbor, MI 48109 and \\ ${ }^{2}$ Department of Radiology, Medical College of Virginia, Virginia Commonwealth University, \\ Richmond, VA 23298 , U.S.A.
}

(Received 10 March 1982; received for publication 17 August 1982)

\begin{abstract}
The medical records, conventional radiographs, bone scans and pelvic CT scans obtained in 50 consecutive patients with malignant lesions involving the osseous pelvis were retrospectively reviewed. In 21 patients with primary bone tumors or with osseous involvement from adjacent pelvic neoplasms, CT provided additional information, in 17 patients $(80 \%)$ which had direct bearing in the clinical management. CT was less useful in 29 patients with bony metastases. Although more information was obtained, this was of clinical significance in only 12 patients.
\end{abstract}

Computed tomography-pelvis Malignant neoplasms, bone

\section{INTRODUCTION}

Malignant lesions involving the osseous pelvis are sometimes difficult to evaluate if not occasionally overlooked by conventional radiography. This is due to the peculiar anatomy of the pelvic bones and its frequent obscuration by overlying bowel contents.

On the basis of its cross sectional display and ability for fine delineation of bone and soft tissue densities, computed tomography (CT) appears particularly well suited to image the bony pelvis and its abnormalities [1-7]. This retrospective review attempts to assess the impact of CT on the diagnosis and management of malignant lesions of the bony pelvis.

\section{MATERIALS AND METHODS}

The medical records and the CT scans obtained in 50 consecutive patients with malignant tumors involving the osseous pelvis were retrospectively reviewed. Plain films, conventional tomograms and radionuclide bone scans in these patients were also evaluated. There were 17 females and 33 males with ages ranging from 12 to $78 \mathrm{yr}$ (mean age, $43 \mathrm{yr}$ ).

CT examinations were performed with a Delta 50 FS or a GE CT/T 8800 total body scanners. All images were analyzed with appropriate gray scale settings for bone in addition to the regular soft tissue display.

Consecutive scans of the entire pelvis were obtained in all patients with $5-12 \mathrm{~mm}$ collimation depending on the clinical indications and type of scanner used. Most scans were performed in the supine position with occasional prone examinations to decrease discomfort in patients with painful lesions. Oral and intravenous contrast material was given to most patients mainly to evaluate intrapelvic extensions and relationship to vascular structures and pelvic organs.

The impact of CT scanning was arbitrarily graded as follows: no additional information: 0 points; additional information, but without influence in patient management: 1 point; additional information with influence in patient management: 2 points (includes: determinations of surgical resectability, alteration of radiation therapy ports, alteration of chemotherapy protocols, CT guided biopsy with positive results); demonstration of a lesion unsuspected prior to CT: 3 points.

Address reprint requests to: Marco A. Amendola, Department of Radiology, Box 13, University Hospital, Ann Arbor, MI 48109. U.S.A. 
Table 1

\begin{tabular}{lcl}
\hline \multicolumn{1}{c}{ Primary tumors } & Cases & Score (points) \\
\hline Osteosarcoma & 3 & $6(2,2,2)$ \\
Chondrosarcoma & 3 & $6(2,2,2)$ \\
Chordoma & 2 & $3(2,1)$ \\
Ewing's sarcoma & 1 & 2 \\
Lymphoma & 1 & 2 \\
$\quad 10$ & 19 (Average 1.9) \\
\hline
\end{tabular}

\section{RESULTS}

Ten patients had primary pelvic bone tumors (Table 1). Direct extension from extraosseous pelvic malignancy was found in eleven patients (Table 2). In 29 patients CT demonstrated metastatic neoplasm to the osseous pelvis (Table 3 ).

According to our grading method CT scans had the highest score in those patients with direct extension from extraosseous pelvic malignancy: 2.09 points. Primary tumors had an average of 1.9 , and distant metastases 1.3 points.

Analysis of Tables 1 and 2 shows that in all patients with primary osseous tumors and in those with bony involvement from adjacent neoplasms, CT provided additional information. This had a direct bearing in the patient clinical management in $80 \%(16 / 21)$. CT was less useful in patients with osseous metastases. More information was obtained in $72 \%(21 / 29)$. However, this was of clinical significance in only $41 \%(12 / 29)$.

Table 2. Direct extension from extraosseous pelvic malignancy

\begin{tabular}{lcc}
\hline & Cases & \multicolumn{1}{c}{ Score } \\
\hline Soft tissue sarcoma & 4 & $7(2,2,2,1)$ \\
Rectum CA & 3 & $7(3,3,1)$ \\
Bladder CA & 2 & $5(3,2)$ \\
SCE cervix & 2 & $4(3,1)$ \\
$\quad 1$ & 23 (Average 2.09) \\
\hline
\end{tabular}

Table 3

\begin{tabular}{lcl}
\hline \multicolumn{1}{c}{$\begin{array}{c}\text { Bone metastases } \\
\text { from }\end{array}$} & Cases & Score \\
\hline Prostate & 5 & $5(2,1,1,1,0)$ \\
Bladder & 3 & $6(3,2,1)$ \\
Lung & 3 & $5(3,2,0)$ \\
Melanoma & 3 & $5(2,2,1)$ \\
Unknown primary & 2 & $3(2,1)$ \\
Lymphoma & 2 & $1(1,0)$ \\
Hypernephroma & 2 & $0(0,0)$ \\
Colon & 1 & 3 \\
Rhabdomyosarcoma & 1 & 3 \\
Penis & 1 & 3 \\
SCE Bartholin Gland & 1 & 2 \\
Liposarcoma & 1 & 1 \\
Ovary & 1 & 1 \\
Breast & 1 & 0 \\
Neuroblastoma & 1 & 0 \\
Undiff CA & 1 & 0 \\
$\quad$ Total & 29 & 38 (Average 1.31)
\end{tabular}


Considering the entire group, CT had a significant impact in patient management in $60 \%(30 / 50)$ of the patients.

Radionuclide bone scans demonstrated abnormal uptake in 20 of 22 patients $(91 \%)$ in which CT evidence of bony involvement was present. The two false negative studies occurred in a patient with recurrent bladder carcinoma with erosion of adjacent bone (Fig. 3), and in another patient with a small lytic lesion of the pubis from Burkitt's lymphoma.

Conventional tomography was obtained in 5 of the 10 patients with primary osseous malignancies. In 3 of these, CT scans were definitely more informative regarding soft tissue mass and extension of tumor across sacro-iliac joints. Angiography and ultrasonography were seldom employed and no comparison will be attempted.

Case 1

\section{ILLUSTRATIVE CASE REPORTS}

A 13-yr old male presented with a large painless mass in the left lower quadrant. The patient had a rhabdomyosarcoma of the left lumbosacral area resected at age two. Surgery was followed by radiation therapy to the tumor bed and systemic chemotherapy. He had been without evidence of disease until the present admission. Plain radiographs of the pelvis revealed a large osteoblastic lesion in the right ileum with questionable involvement of the left sacrum, consistent with a radiation induced osteogenic sarcoma (Fig. 1A). Subsequent ${ }^{99} \mathrm{mTc}-$ MDP bone scan demonstrated a large area of increased uptake in the left hemipelvis, however sacral involvement could not be ruled out (Fig. 1B). CT scan demonstrated a sharp cleavage plan still present between the tumor and the left sacral wing, in addition to accurate delineation of the intrapelvic extension of the tumor (Fig. 1C). Extensive work-up failed to show evidence of distant metastases. A successful left hemipelvectomy was performed confirming the CT findings.

\section{Case 2}

A 28-yr old male noticed a sharp pain in his left buttock about 3 months prior to his current admission, due to increasing pain and appearance of a palpable mass in the area.

Percutaneous needle biopsy revealed undifferentiated soft tissue sarcoma. Chest radiographs showed metastatic lung disease. CT scan of the pelvis to evaluate local extent of the disease showed a $20 \mathrm{~cm}$ soft tissue mass involving the gluteal muscles from the iliac crest to the acetabulum (Fig. 2). Evidence of bone invasion at the posterior aspect of the left ileum was noted. Radiation therapy ports were outlined according to the CT information.

\section{Case 3}

A 62-yr old male had a previous history of partial cystectomy and chemotherapy for squamous cell carcinoma arising in a bladder diverticulum.

A right pelvic mass displacing the bladder to the left and causing right sided ureteral obstruction was noted at urography (Fig. 3A). No evidence of bony involvement was present in plain films or radionuclide bone scan. A CT scan for therapy planning demonstrated invasion of the medial supra-acetabular portion of the right ileum (Fig. 3B).

\section{Case 4}

A 48-yr old male status post penile resection for squamous cell carcinoma had a CT scan of the abdomen and pelvis for evaluation of tumor spread. This study disclosed a $1 \mathrm{~cm}$ solitary lytic lesion in the left ischium, substantiated in plain radiographs of the pelvis obtained after the CT scan diagnosis (Fig. 4A-C). Follow-up films of the pelvis showed significant increase in lesion size 3 months later, despite chemotherapy, consistent with a growing metastatic lesion (Fig. 4D).

Case 5

A 70-yr old female was admitted to the hospital with a 6 week history of progressive weakness and numbness of the legs more marked on the right side. Urinary incontinence had developed in the week prior to admission. Past history included a total abdominal hysterectomy for adenocarcinoma of the uterus $13 \mathrm{yr}$ previously without known recurrence. In the initial work-up lumbosacral spine 


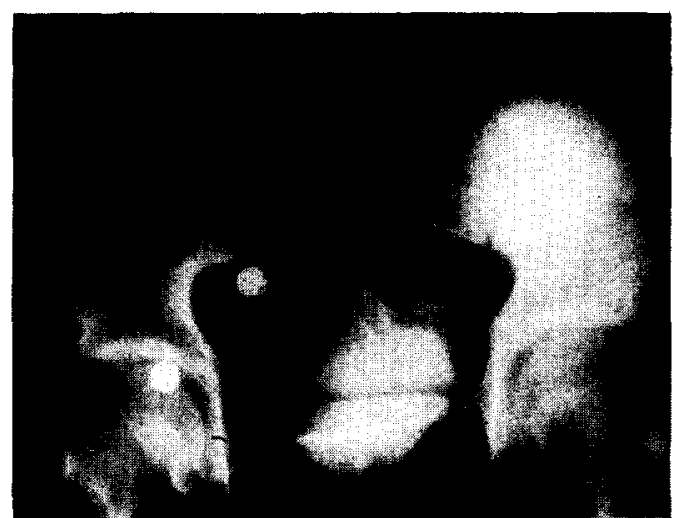

(A)

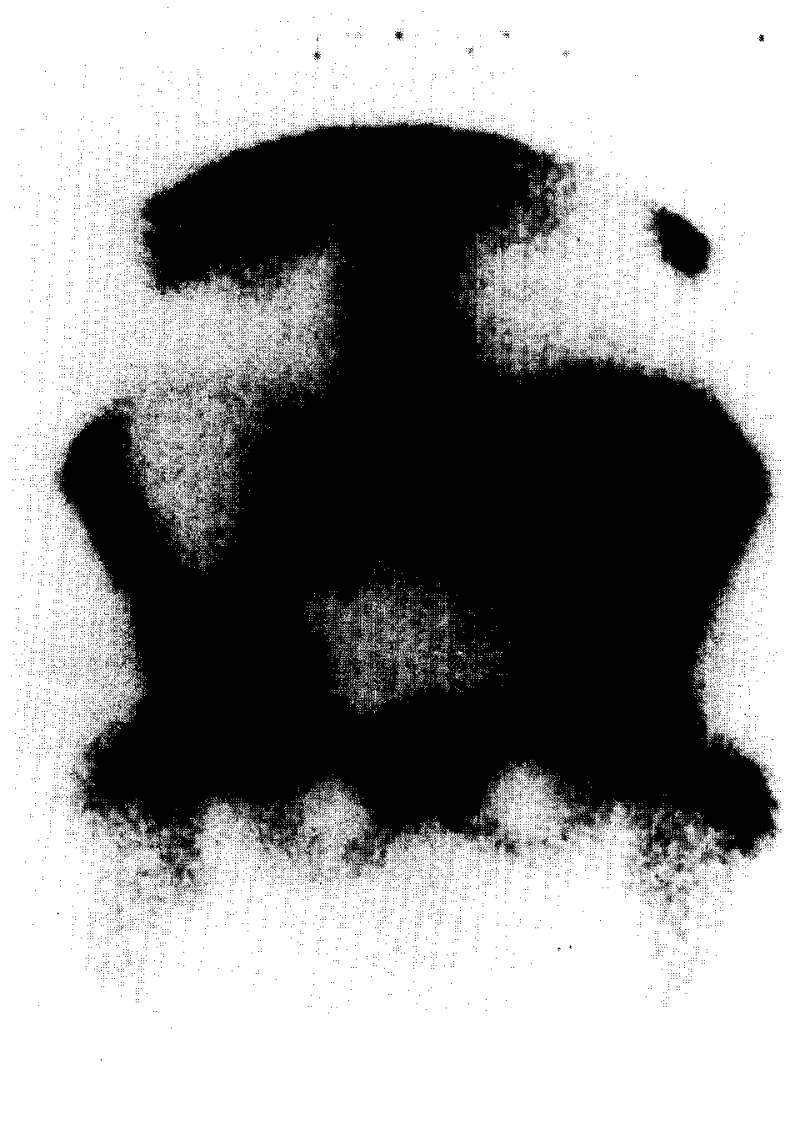

(B)

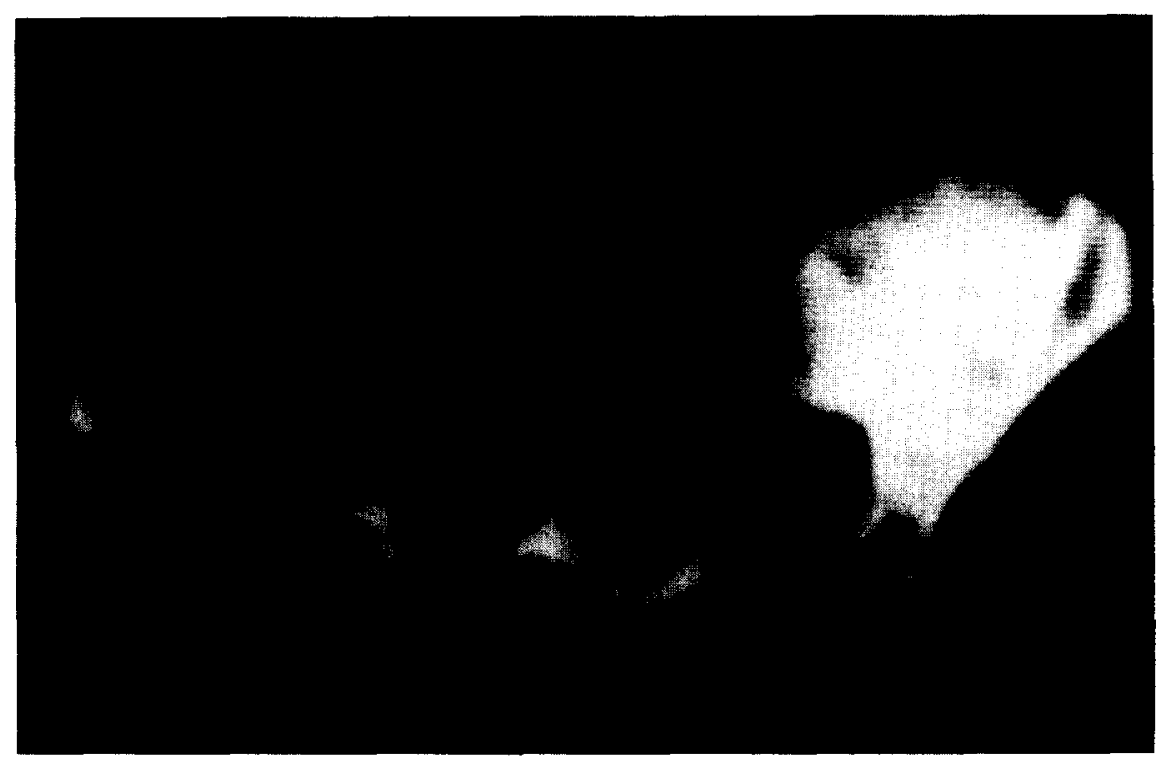

(C)

Fig. 1.(A) AP radiograph of the pelvis: large osteoblastic lesion of left ileum with possible sacral involvement, consistent with radiation induced osteosarcoma. Note the smaller size of left iliac wing and scoliosis secondary to radiation. Contrast in rectosigmoid and right ureter from previous studies. At CT left ureter was displaced medially but not involved by the tumor (not shown). (B) Radionuclide bone scan: increased uptake in left hemipelvis, sacral involvement cannot be excluded. (C) CT scan: large intrapelvic component of blastic lesion arising from left ileum. Left sacral wing is free of tumor at this, as well as in other levels. Successful hemipelvectomy performed. 


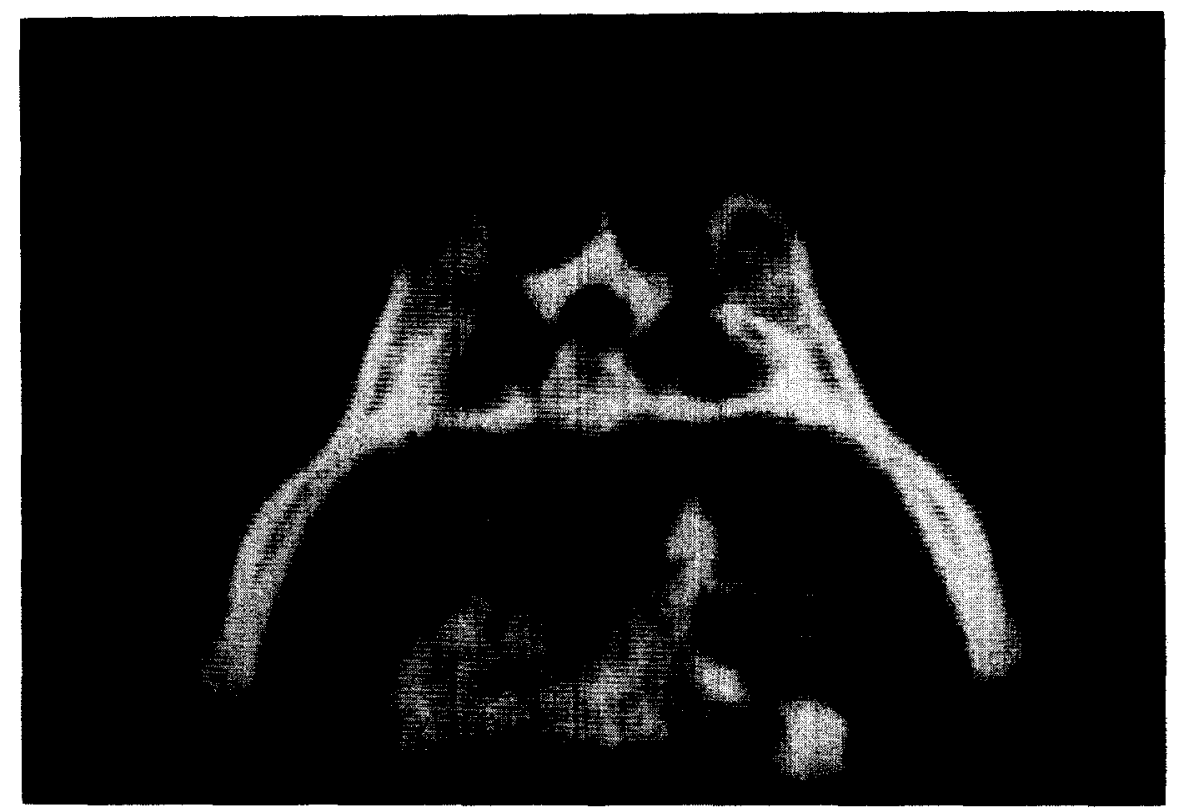

Fig. 2. Homogenously massive enlargement of left gluteal muscles and bone erosion at posterosuperior iliac spine. The sacrum remains intact and no intrapelvic extension is demonstrated. Patient started on radiation therapy for undifferentiated soft tissue sarcoma (CT scan obtained in the prone position).

radiographs were interpreted as showing degenerative changes at L5-S1 apophyseal joints and sacroiliac joints without evidence of bone destruction. Pelvic ultrasonography was negative. A CT scan of the abdomen and pelvis disclosed an extensive destructive lesion of the right side of the sacrum with partial involvement of the left sacral wing (Fig. 5).

Also numerous irregular low density lesions within the liver parenchyma were felt to be indicative of metastatic disease. Subsequent metrizamide myelography demonstrated an irregular, largely circumferential extradural encroachment upon the caudal sac with obliteration of the sacral nerve root sleeves distal to the L5-S1 space presumably secondary to extension from the sacral neoplasm. A sacral bone biopsy showed a poorly differentiated adenocarcinoma. The patient was referred to radiation therapy and after $\mathbf{4 4 0 0}$ rad to the sacral area partial symptomatic relief was obtained.

\section{DISCUSSION}

Problems in the diagnostic imaging of the osseous pelvis are partly due to its complex anatomy. The sacral curvature, oblique orientation of the sacroiliac joints, sigmoid shape of iliac bones and foreshortening of ischiopubic rami, militate against its accurate display by conventional radiographic and tomographic projections [3]. The cross sectional view afforded by CT helps unravel superimposed bony structures. Superimposition of the sacral wing and the posterosuperior iliac bone creates difficulty in the correct assignment of pathologic processes in this area on routine radiographs. Our experience is in agreement with other authors regarding the superiority of CT in delineating abnormalities adjacent to the sacroiliac joints $[3,5-8]$.

Obscuration of lesions of sacrum and ileum by overlying bowel contents in pelvic X-rays and by bladder activity in radionuclide bone scans is obviated by CT. Soft tissue masses associated with pelvic malignancies are directly and precisely imaged by CT. In our study this translated into very specific determination of tumor margins which facilitated radiation therapy planning.

In our series of primary osseous malignancies originating in the pelvic girdle, the specific diagnosis was either available from previous biopsy or strongly suggested from conventional radiographic studies prior to CT. However in 9 of 10 patients in this group, the ability of CT to determine precise extension of tumor was instrumental in affecting therapeutic management. Decisions regarding the feasibility and scope of surgical procedures were greatly aided. CT demonstration of extent 


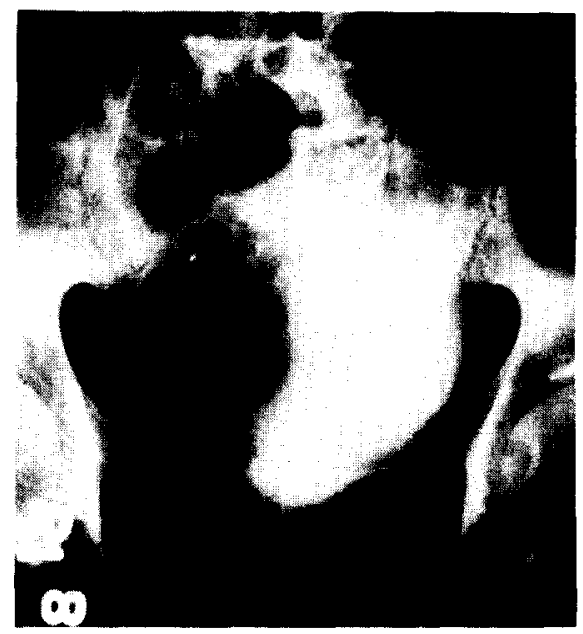

(A)

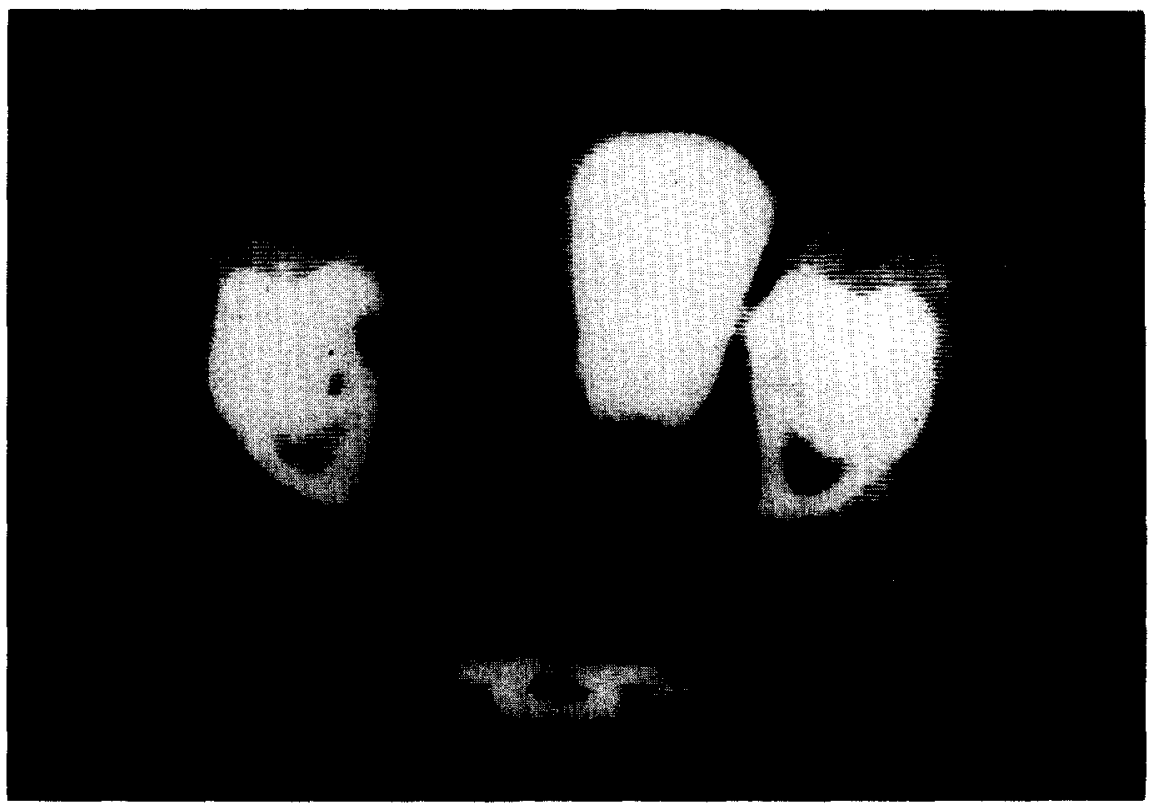

(B)

Fig. 3.(A) Coned down views of the pelvis during intravenous urogram: tumor mass of recurrent carcinoma invading and displacing bladder. No pelvic osseous involvement present in AP or oblique views of the pelvis. (B) Unquestionable bone erosion at medial aspect of supra-acetabular iliac bone demonstrated by CT.

and anatomic relationships of soft tissue components of osseous neoplasms was used to advantage in outlining radiation therapy ports, for optimization of treatment planning (Fig. 6).

A large number of patients with primary malignancies of the pelvic organs are examined with $C T$ for staging, follow-up and evaluation of recurrent disease [9-13]. CT has shown in several instances direct extension of disease to the osseous pelvis not demonstrated in conventional X-rays or even clinically silent. This had a significant impact on stage upgrading and on the general oncologic management of these patients. In this regard the need for analysis of the CT image using proper window widths and levels for bone, in addition to the usual soft tissue display cannot be overemphasized [1]. Routine application of this principle has enabled us to diagnose unsuspected bony invasion in patients with soft tissue tumors (Fig. 2) and pelvic organ malignancies (Fig. 3). CT also discovered metastatic lesions to the pelvic skeleton undisclosed by other imaging modalities (Figs 3 , 5). Skeletal scintigraphy is the most sensitive method currently available for the early detection of bone metastases $[14,15]$. The high sensitivity of radionuclide scanning was again shown in our 


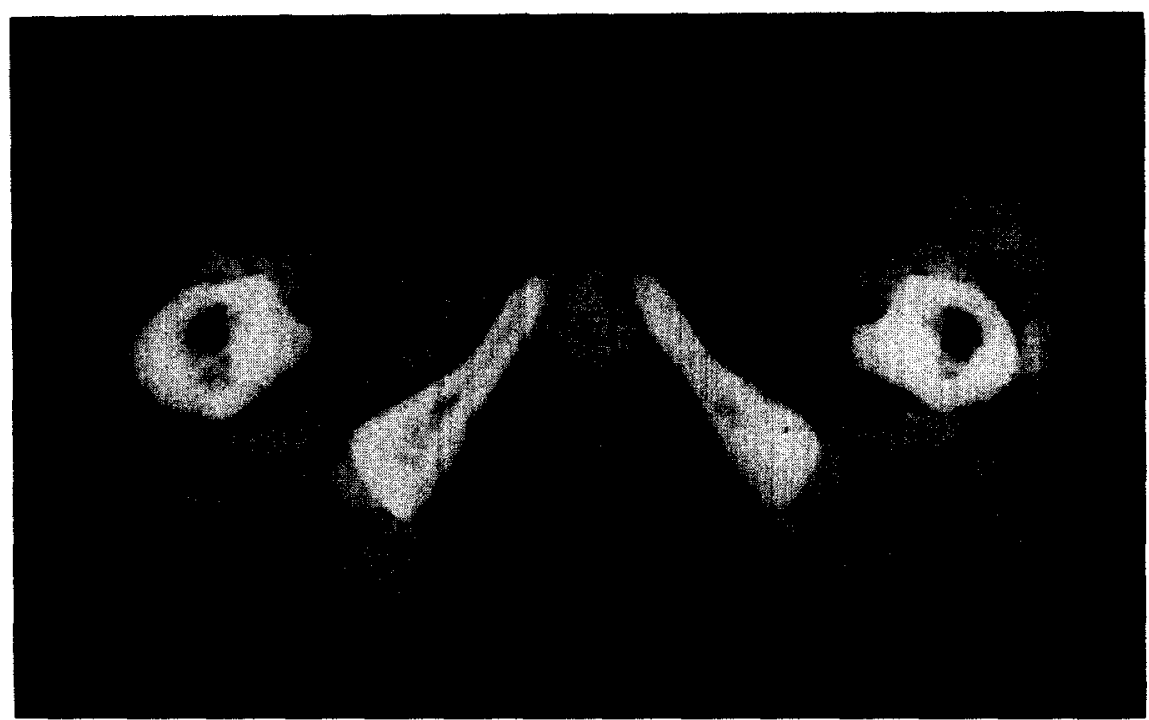

(A)

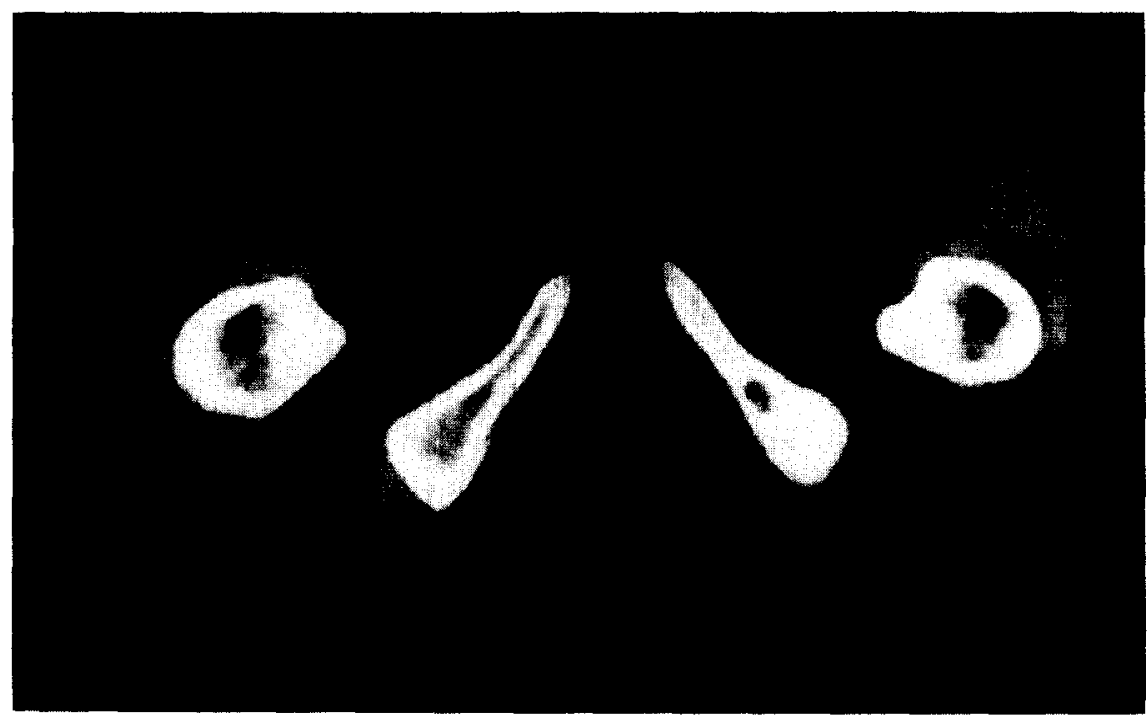

(B)

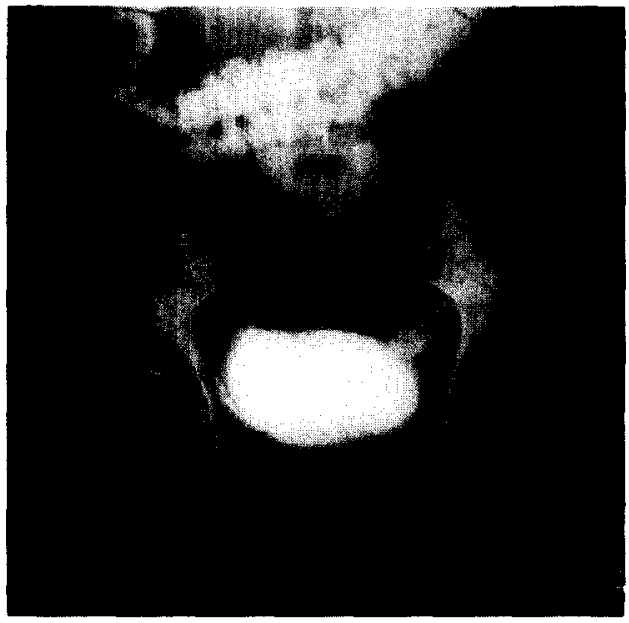

(C)

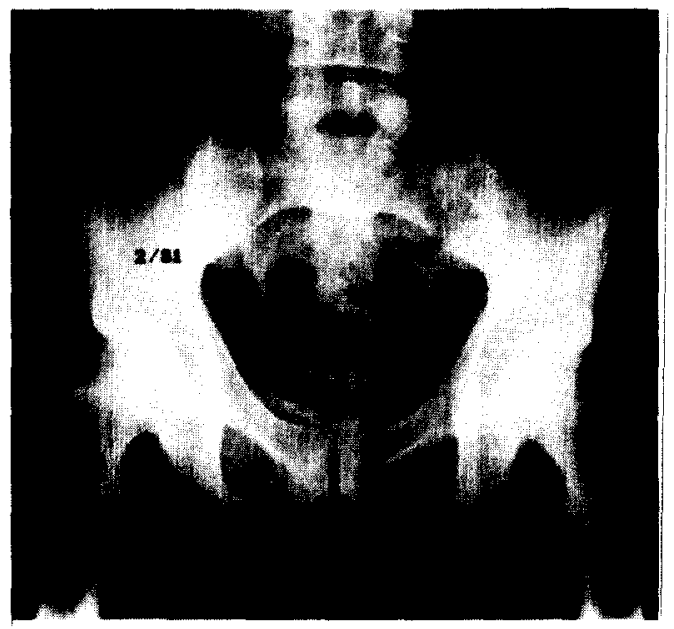

(D)

Fig. 4.(A, B) Small lytic lesion in left ischium, better secn on narrow window settings. (C) AP radiograph of pelvis confirms CT findings. (D) Despite intensive chemotherapy plain film of the pelvis demonstrates marked increase in size consistent with a growing metastatic lesion. 


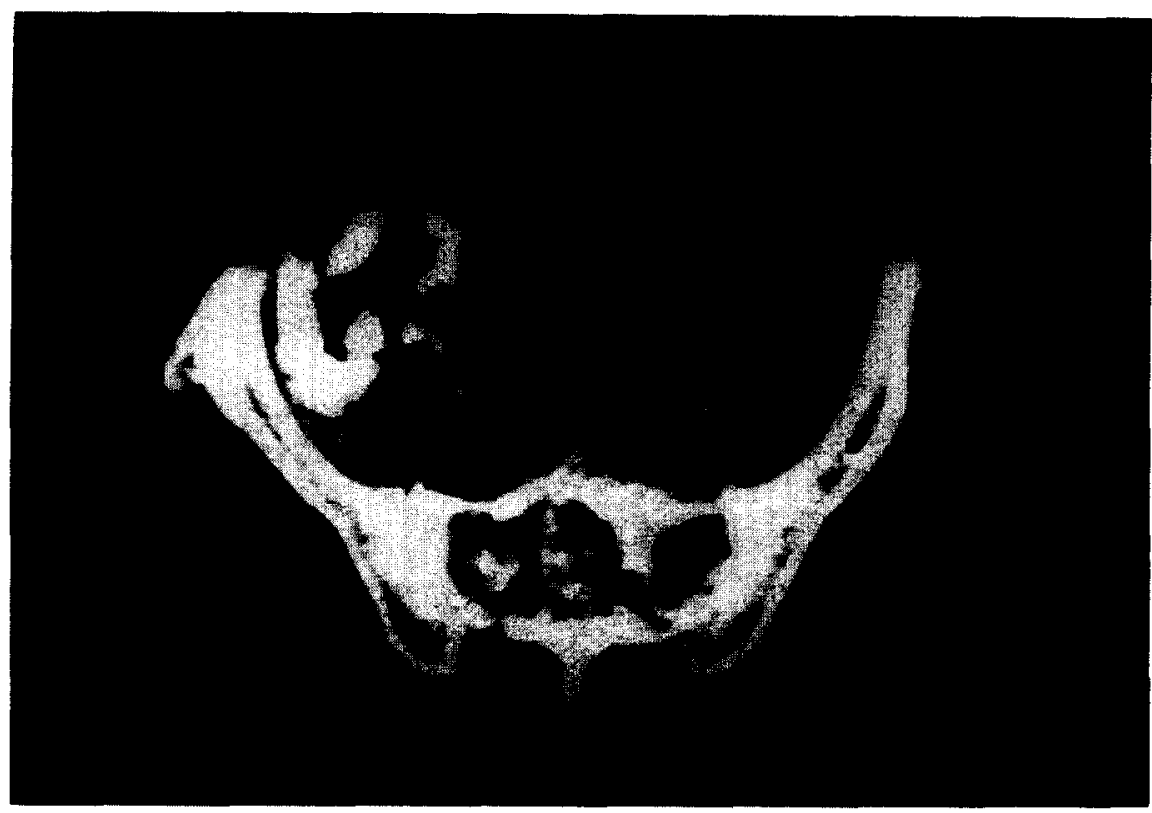

Fig. 5. Extensive sacral lesion demonstrated by CT scan obtained within one week time of plain radiographs negative for pelvic osseous malignancy.

series $(91 \%)$ confirming it as the screening modality of choice in the diagnostic work-up of patients suspected of metastatic bone disease. Bone scans are however, nonspecific and increased uptake may be produced by Paget's disease, arthritis, trauma and other nonmalignant conditions.

Correlation with radiographs of areas of increased uptake or symptomatic sites is recommended to avoid false results $[16,17]$. In 3 of our patients conventional X-rays did demonstrate arthritic changes at the site of abnormally increased uptake but no neoplastic involvement. However, subsequent CT documented definite bone destruction. There were two patients with negative bone scans and radiographs in which CT demonstrated small lytic lesions (Fig. 3). False negative bone scans have been described with purely lytic lesions, and those smaller than the resolution of the current imaging instruments [17-19]. The potential value of CT in selected patients with lumbosacral neuropathy has also recently been described [20,21]. Although we do not recommend CT of the pelvis for primary evaluation of metastatic bone lesions the value of CT in equivocal circumstances should be recognized.

\section{SUMMAR Y}

A retrospective review of pelvic CT scans and medical records of 50 consecutive patients with malignant tumors involving the osseous pelvis was performed. Ten patients had primary pelvic bone tumors: Osteosarcoma, chondrosarcoma, Ewing's tumor, chordoma. Eleven patients had direct osseous extension from other pelvic malignancies: carcinoma of the bladder, cervix, rectum. Twentynine patients had metastatic lesions to the bony pelvis from bladder, prostate, lung, melanoma, breast and other primary tumors.

The impact of CT scanning was arbitrarily graded as follows: no additional information: 0 points; additional information, but without influence in patient management: 1 point; additional information with influence in patient management: 2 points (includes: determination of surgical resectability, alteration of radiation therapy ports, alteration of chemotherapy protocols, CT guided biopsy with positive results); Demonstration of lesions unsuspected prior to CT: 3 points.

In all patients with primary osseous tumors and with osseous involvement from adjacent pelvic neoplasms, CT added information, which had direct bearing in the patient's clinical management in $80 \%(17 / 21=80 \%)$. 
(A)

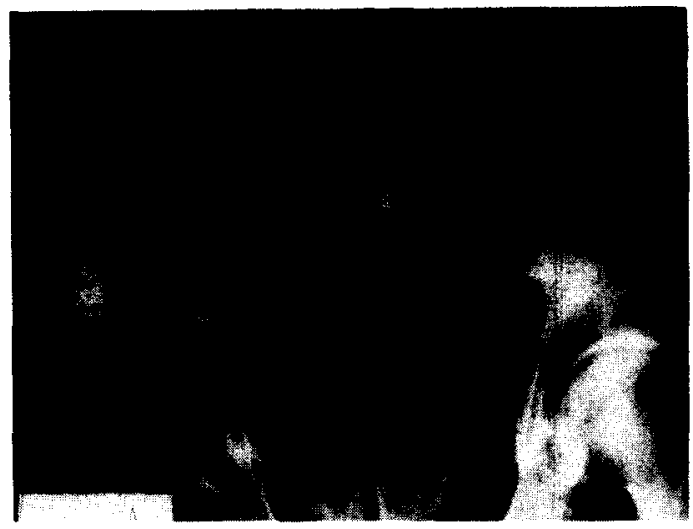

(B)
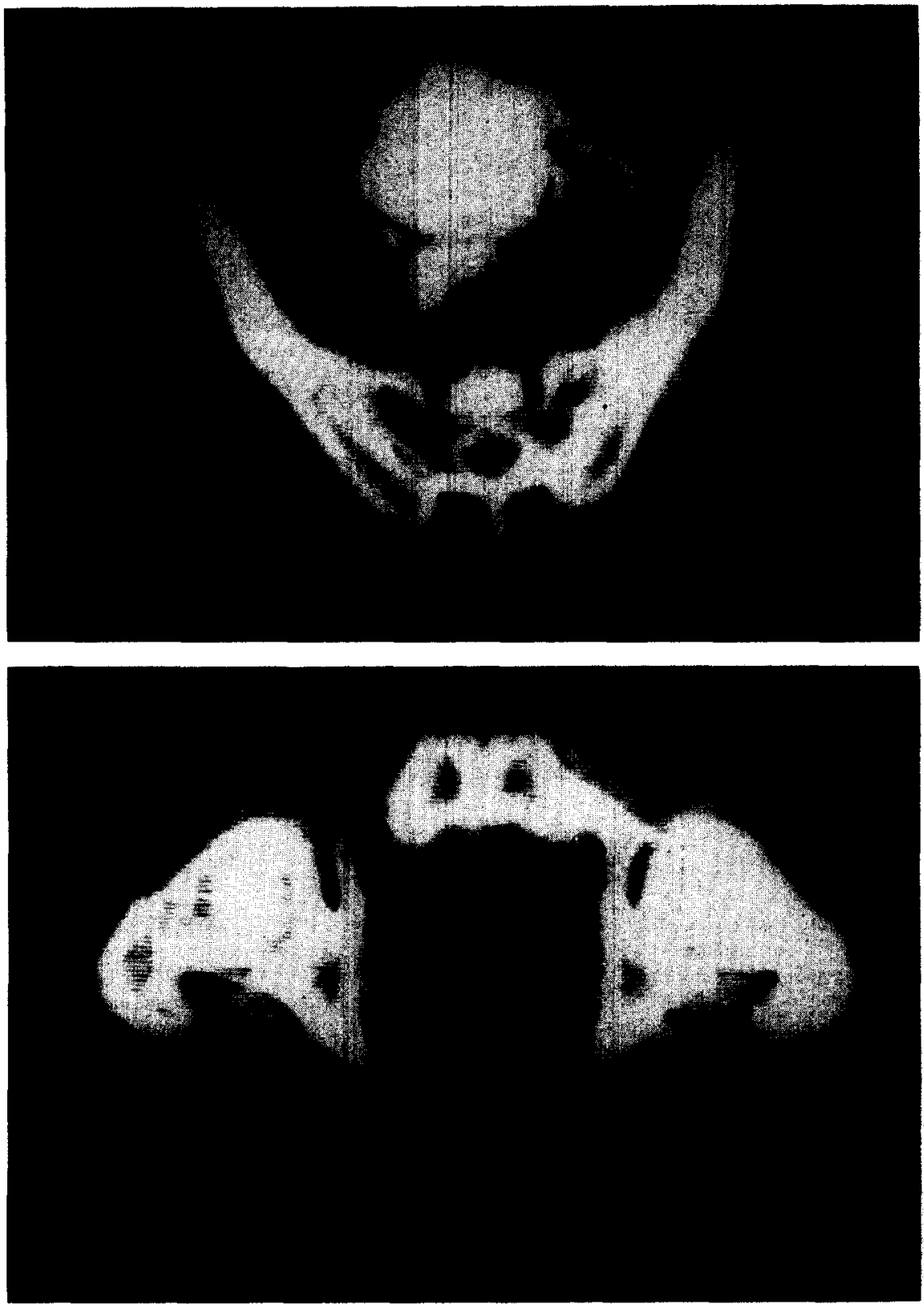

(C)

Fig. 6.(A-C) Sacral chordoma in 66-yr old male (A) AP radiograph: Destructive expansile lesion with associated mass. Complete extent of soft tissue component, and relationship to contrast filled bladder and rectosigmoid best demonstrated by $C T$. Note small calcifications in lower portion of the mass $(B, C)$. 
CT was less useful in patients with bony metastases. More information was obtained in $72 \%$ $(21 / 29)$, but this was of significance in only $41 \%$.

Acknowledgement-The authors wish to express their appreciation to Ms Juanita Cantrell for expert secretarial help.

\title{
REFERENCES
}

1. R. A. Mcleod, D. H. Stephens, J. W. Beabout et al., Computed tomography of the skeletal system, Semin. Roentg. 13, 235-247 (1978).

2. L. Weis, R. T. Heelan and R. C. Watson, Computed tomography of orthopedic tumors of the pelvis and lower extremities, Clin. Orthop. 130, 254-259 (1978).

3. L. A. Gilula, W. A. Murphy, C. C. Tailor et al., Computed tomography of the osseous pelvis, Radiology 132, 107-114 (1979).

4. J. S. Wilson, M. J. Korobkin, H. K. Genant and E. G. Bovill, Computed tomography of musculoskeletal disorders, $A m$. J. Roentg. 131, 55-61 (1978).

5. L. A. deSantos, H. M. Goldstein, J. A. Murray et al., Computed tomography in the evaluation of musculoskeletal neoplasms, Radiology 128, 89-94 (1978).

6. P. E. Berger and J. P. Kuhn, Computed tomography of tumors of the musculoskeletal system in children, Radiology 127 , 171-175 (1978).

7. E. Levine, K. R. Lee, J. R. Neff et al., Comparison of computed tomography and other imaging modalities in the evaluation of musculoskeletal tumors, Radiology 131, 431-437 (1979).

8. G. F. Carrera, W. D. Foley, F. Kozin et al., CT of sacroiliitis, Am. J. Roentg. 136, 41-46 (1981).

9. M. A. Amendola, J. W. Walsh, B. E. Amendola et al., Computed tomography in the evaluation of carcinoma of the ovary, J. Comput. assist. Tomogr. 5, 179-186 (1981).

10. J. W. Walsh, M. A. Amendola, D. J. Hall et al., Recurrent carcinoma of the cervix: CT diagnosis, Am. J. Roentg. 136, $117-122(1981)$

11. J. 'Tisnado, M. A. Amendola, J. W. Walsh et al., Computed tomography of the perineum, Am. J. Roentg. 136, 475-481 (1981).

12. J. W. Walsh, M. A. Amendola, K. F. Konerding et al., Computed tomographic demonstration of pelvic and inguinal node metastasis from primary and recurrent pelvic malignancy, Radiology 137, 157-166 (1980).

13. M. A. Amendola, B. E. Amendola, T. A. Hazra et al., Body computed tomography in the evaluation of pediatric malignancy. Computerized Tomogr. 5, 219-228 (1981).

14. C. B. Galasko, Mechanism of uptake of bone imaging isotopes by skeletal metastases, Editorial, Clin. Nucl. Med. 5 , $565-568$ (1980).

15. R. E. O'Mara, Skeletal scanning in neoplastic disease, Cancer 37, $480-486$ (1976)

16. J. C. Mall, C. Bekerman, P. B. Hoffer et al., A unified radiological approach to the detection of skeletal metastases, Radiology 118, 323-328 (1976).

17. C. B. Galasko, Problems associated with the detection of skeletal metastases, J. R. Soc. Med. 71, 38-41 (1978).

18. H. D. Covelli, A. J. Zaloznik and K. M. Shekitka, Evaluation of bone pain in carcinoma of the lung. Role of the localized false-negative scan, J. Am. Med. Assoc. 244, 2625-2627 (1980).

19. R. K. Loeffler, R. N. DiSimone and W. J. Howland, Limitations of bone scanning in clinical oncology, J. Am. Med.Assoc 234, 1228-1232 (1975)

20. M. P. Federle, A. A. Moss and F. R. Margolin, Role of computed tomography in patients with "sciatica", J. Comput. assist Tomogr. 4, 335-341 (1980).

21. G. Di Chiro and D. Schellinger, Computed tomography of spinal cord after lumbar intrathecal introduction of metrizamide (Computed Assisted Myelography), Radiology 120, 101-104 (1976).

\begin{abstract}
About the Author-Marco A. Amendola is currently an Associate Professor of Radiology at the University of Michigan. He received his M.D. degree from the School of Medicine, University of Uruguay, Montevidco. His residency in Radiology and fellowship in Computed Tomography were completed at Albert Einstein Medical Center, Philadelphia, Pennsylvania. He is Director of Genito-Urinary Radiology and Co-Director of Abdominal Radiology Division at the University of Michigan Medical Center in Ann Arbor, Michigan.
\end{abstract}

About the Author-Khalil ShIRaZI received his M.D. degree from the University of Mashhad, Iran. His residencies in General Radiology, and Diagnostic Radiology were done at Sorbonne University, Paris, France; Tulane University, New Orleans; and Temple University, Philadelphia, Pennsylvania. He has held faculty positions in Radiology at Duke University Medical Center, Medical College of Virginia; and University of Michigan. Currently, he is Acting Director of Chest Radiology at the University of Michigan Medical Center, Ann Arbor, Michigan.

About the Author-Beatriz E. Amendola received her M.D. degree from the School of Medicine, University of Uruguay, Montevideo, in 1974. She received her training in Radiation Oncology at the Medical College of Virginia, Virginia Commonwealth University. She is presently Assistant Professor of Radiology and Acting Chief of the Radiation Therapy Department at the University of Michigan Medical Center, Ann Arbor, Michigan. 
About the Author-Lawrence R. Kuhns received his M.D. degree from the School of Medicine, University of Washington, Seattle Washington in 1969. His residency in Pediatric Radiology was completed at the University of Michigan. He is currently attending Radiologist at St Joseph Mercy Hospital, Ann Arbor, Michigan and Clinical Professor of Radiology at the University of Michigan Medical Center, Ann Arbor, Michigan.

About the Author-Jaime TiSnado received his M.D. degrce from San Marcos National University, Lima, Peru. His Radiology residency and fellowship in angiography were done at Thomas Jefferson University Hospital in Philadelphia, Pennsylvania. Currently, he is Associate Professor of Radiology and Director of the Cardiovascular Section of the Department of Radiology at the Virginia Commonwealth University Medical College of Virginia Hospitals.

About the Author-Issa Yaghmal earned his M.D. degree from Teheran University in 1959. He completed his radiology training at the Illinois Masonic and Cook County Hospitals in Chicago. He has held faculty positions at the University of Teheran and since 1973 at the Medical College of Virginia, Virginia Commonwealth University where Dr Yaghmai is presently Professor of Radiology and Director of Skeletal Radiology. 